\title{
Experimental and Numerical Study of the Sloshing Modes of Liquid Storage Tanks with the Virtual Mass Method
}

\author{
G. YAZICI ${ }^{a, *}$, S.A. KILIÇ ${ }^{b}$ AND K. ÇAKIROĞLU ${ }^{b}$ \\ ${ }^{a}$ Istanbul Kultur University, Department of Civil Engineering, Istanbul, Turkey \\ ${ }^{b}$ Bogazici University, Department of Civil Engineering, Istanbul, Turkey
}

\begin{abstract}
Accurate prediction of the vibration characteristics of the sloshing motion is essential for the analysis and design of liquid storage tanks subjected to base motion. The virtual mass method is a computationally efficient approach to determine the hydrodynamic forces generated by incompressible and inviscid fluids in accelerated containers since the virtual mass method involves meshing of the fluid boundaries rather than the entire fluid domain. Hydrodynamic actions of the sloshing liquid are taken into consideration by coupling a virtual fluid mass matrix to the structural points on the wetted regions of the tank wall. Analysis of the free surface displacements of the contained liquid can be carried out using the virtual mass method and this paper focuses on the application of the virtual mass method for the analysis of the vibration frequencies and mode shapes of the sloshing modes in rectangular and cylindrical liquid storage tanks. Firstly, the theoretical background for the analytical solution of the mode shapes and modal frequencies is presented for rectangular and cylindrical liquid tanks. This is followed by the description of the procedure used to apply the virtual mass method to obtain the sloshing modes and mode shapes of the contained liquid. The effects of various surface mesh topologies on the predicted vibration characteristics are compared with the analytical solutions as well as the results of an experimental study conducted on scaled rectangular and cylindrical containers mounted on a shake table.
\end{abstract}

DOI: 10.12693/APhysPolA.135.1068

PACS/topics: virtual mass method, meshing, liquid storage tanks, sloshing, fluid structure interaction

\section{Introduction}

Liquid storage tanks are critical components of environmental engineering and petrochemical facilities. Field reports from past earthquakes indicate that these structures are quite susceptible to earthquake-induced damage due to hydrodynamic forces generated by sloshing, the violent shaking of the contained liquid due to the movement of the tanks [1]. Sloshing depends on a variety of factors including the tank geometry, liquid depth and the characteristics of the external excitation [2]. Accurate estimate of the vibrational characteristics of the sloshing motion is vital for the analysis and design of liquid storage tanks and considerable research has been conducted on the analysis of the response of partially filled liquid containers subjected to acceleration. A thorough review of the analytical and numerical methods can be obtained from [3-5]. This paper focuses on the application of the virtual mass method for the analysis of the vibration frequencies and mode shapes of the sloshing modes in rectangular and cylindrical liquid storage tanks.

\section{Theoretical background}

Sloshing wave motion will be assumed to be linearly proportional to the amplitude of the base motion and potential flow theory, which is widely used in sloshing problems in non-shallow liquids, will be used to calculate

*corresponding author; e-mail: g.yazici@iku.edu.tr the sloshing displacements. Assuming the flow is inviscid, irrotational and incompressible, the velocity distribution within the sloshing liquid can be approximated by using a velocity potential function $\Phi$ which satisfies the Laplace equation Eq. (1) throughout the liquid domain. It is convenient to use a stationary and a moving Cartesian coordinate system in the formulation of the liquid surface displacements.

$$
\Phi \nabla^{2}=0 .
$$

Boundary conditions at the fluid-tank interface and at the free surface of the fluid need to be defined in order to obtain the solution of Eq. (1). If the tank wall is considered to be non-deformable, the velocity component of the fluid normal to the fluid-tank interface should be equal to the corresponding velocity component of the tank wall. In the potential flow theory, there are two free surface boundary conditions, namely, the dynamic free surface boundary condition and the kinematic free surface boundary condition. The dynamic free surface boundary condition is derived by equating the pressure at the free surface to zero or atmospheric pressure in the unsteady Bernoulli equation. The kinematic free surface boundary condition is obtained by equating the vertical velocity of the fluid particles on the free surface to the vertical velocity of the free surface. Step by step derivation of the boundary conditions and the solution of the velocity potential function as well as the sloshing mode shapes and their corresponding periods using Cartesian and cylindrical coordinate systems can be obtained from [3] and [4].

The sloshing mode frequencies $\omega_{m n}$ for cylindrical containers in terms of cylindrical coordinates $(r, \theta)$ can be 
obtained from Eq. (2), where $m$ and $n$ are the number of nodal diameters and the nodal circles for a given sloshing vibration mode, $h$ is the height of the liquid, $g$ is the gravitational acceleration, $R$ is the radius of the tank, $J_{m}$ is the Bessel function of the first kind and order $m$ and $\lambda_{m n}=\xi_{m n} / R$ are the roots of $\partial J_{m}\left(\lambda_{m n} r\right) /$ $\left.\partial r\right|_{r=R}=0$ [3]:

$$
\omega_{m n}^{2}=\frac{g \xi_{m n}}{R} \tanh \left(\frac{h \xi_{m n}}{R}\right) .
$$

The sloshing mode shapes for a cylindrical tank can be obtained with the Eq. (2), where $\eta$ is the fluid surface elevation measured from the undisturbed free surface, $\alpha_{m n}$ and $\beta_{m n}$ are the constant coefficients determined from the initial conditions [3]:

$$
\begin{aligned}
\eta & =\frac{1}{g} \sum_{m=0}^{\infty} \sum_{n=1}^{\infty}\left[\alpha_{m n} \cos m \theta+\beta_{m n} \sin m \theta\right] J_{m}\left(\lambda_{m n} r\right) \\
& \times \cosh \left(\lambda_{m n} h\right)\left(\omega_{m n} \cos \omega_{m n} t\right)
\end{aligned}
$$

The sloshing mode frequencies $\omega_{i j}$ for rectangular containers in terms of the Cartesian coordinates $(x, y)$ can be obtained from Eq. (3) [6], where, $L_{1}$ and $L_{2}$ are the plan dimensions of the rectangular tank and $k_{i, j}=$ $\pi \sqrt{\left(i / L_{1}\right)^{2}+\left(j / L_{2}\right)^{2}}$ :

$$
\omega_{i j}^{2}=k_{i, j} \tanh \left(k_{i, j} h\right) \text {. }
$$

The sloshing mode shapes for rectangular containers can be obtained by Eq. (4) [6]:

$$
\begin{aligned}
& f_{i, j}(x, y)=\cos \left(\frac{i \pi\left(x+L_{1} / 2\right)}{L_{1}}\right) \\
& \times \cos \left(\frac{j \pi\left(y+L_{2} / 2\right)}{L_{2}}\right) .
\end{aligned}
$$

\section{Experimental study}

The plexiglass tank models used in the experimental study were filled up to a height of approximately $300 \mathrm{~mm}$ with water and excited harmonically at the base in the horizontal direction with a table-top shake table. The diameter of the cylindrical model was $200 \mathrm{~mm}$. The plan dimensions of the prismatic models were $150 \mathrm{~mm} \times 100 \mathrm{~mm}$ and $200 \mathrm{~mm} \times 200 \mathrm{~mm}$. The height and wall thickness of all models were $500 \mathrm{~mm}$ and $3 \mathrm{~mm}$, respectively. Each experiment was filmed at a frame rate of $25 \mathrm{fps}$. The shooting angle of the camera was directed diagonally towards the top of the tanks which made it possible to observe the liquid surface clearly throughout the experiment. The videos of the experiments were later analyzed to detect and match the mode shapes and their modal frequencies with those obtained from the analytical expressions and the virtual mass models [7].

\section{Numerical study}

The virtual mass method was used to obtain the numerical solution for the sloshing mode shapes and their corresponding frequencies for the tank models used in the experimental study. The virtual mass method used in this study is suited for modeling fluids with a free surface contained within a flexible structure. Numerical models used in this study were created and analyzed using MSC NASTRAN finite element analysis software.

In the virtual mass method, the fluid is represented by a coupled mass matrix attached directly to the structural points. Compressibility effects are neglected and it is assumed that the important frequency range for the structural modes is above the gravity sloshing frequencies and below the compressible acoustic frequencies. It is further assumed that the density within a volume is constant and no viscous or rotational flow effects are considered. The incompressible fluid produces a mass matrix defined with full coupling between accelerations and pressures on the flexible structural boundaries. The Helmholtz method used by the virtual mass method solves the Laplace equation by distributing a set of sources over the outer boundary, each producing a simple solution to the differential equation. By matching the boundary motions to the effective motion caused by the sources, a linear matrix equation is solved for the magnitude of the sources. The values of the sources determine the effective pressures, which are used in return to calculate the forces on the grid points. The matrix equation solved by using the virtual mass method utilizes a dense virtual mass matrix. Surface sloshing effects can be modeled by simulating the gravity effects on the free surface with scalar springs that can only move in the normal direction. The sloshing on the free surface can be visualized by the use of a phantom surface boundary that is constructed from shell finite elements, which have nearly zero stiffness in the normal direction. This approach couples only the fluid displacements in the normal direction; therefore, the computed edge or corner effects and tangential motions are approximate [8].

TABLE I

The deviations of sloshing mode frequencies of virtual mass models from the theoretical values [9].

\begin{tabular}{c|c|c|c|c}
\hline \hline \multirow{2}{*}{ Mode } & \multicolumn{3}{|c}{ Deviation [\%] } \\
\cline { 3 - 5 } & $D=100 \mathrm{~mm}$ & $D=150 \mathrm{~mm}$ & $D=200 \mathrm{~mm}$ \\
\hline 1 & 1 & 3.0 & 9.7 & 20.1 \\
2 & 1 & 2.7 & 7.9 & 16.5 \\
0 & 2 & 1.8 & 3.9 & 8.4 \\
3 & 1 & 4.9 & 9.1 & 16.1 \\
4 & 1 & 7.3 & 10.7 & 16.9 \\
1 & 2 & 3.3 & 4.3 & 8.4 \\
5 & 1 & 4.2 & 4.9 & 8.6 \\
2 & 2 & 5.2 & 5.5 & 8.8 \\
0 & 3 & 7.0 & 5.7 & 8.9 \\
3 & 2 & 7.5 & 7.1 & 10.2 \\
1 & 3 & 6.5 & 6.5 & 9.1 \\
6 & 1 & 14.7 & 16.6 & 21.2 \\
4 & 2 & 10.6 & 9.3 & 11.9 \\
2 & 3 & 9.6 & 8.1 & 10.2 \\
1 & 4 & 4.3 & 5.5 & 10.1
\end{tabular}




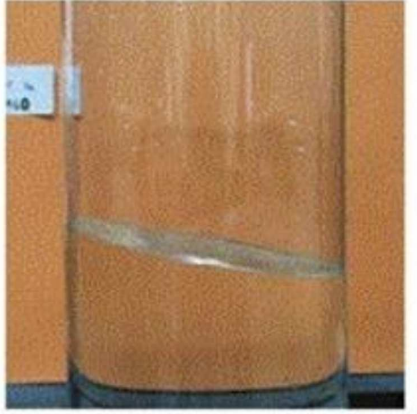

(a)

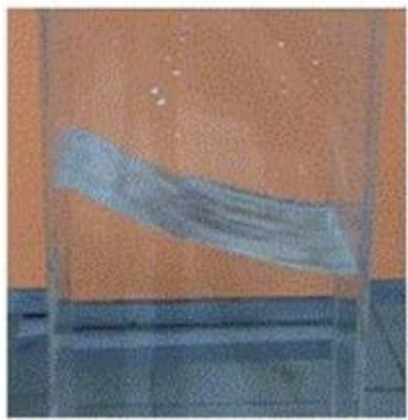

(e)

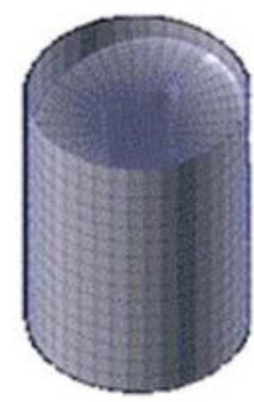

(b)

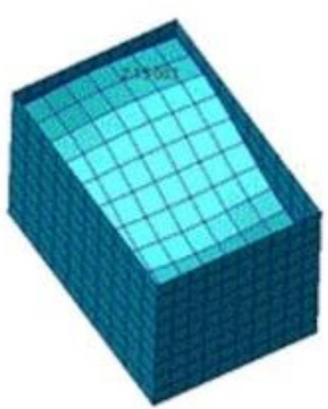

(f)

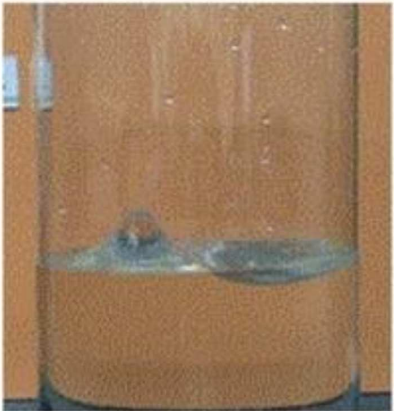

(c)

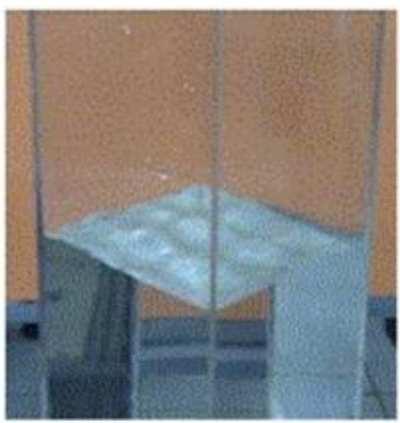

(g)

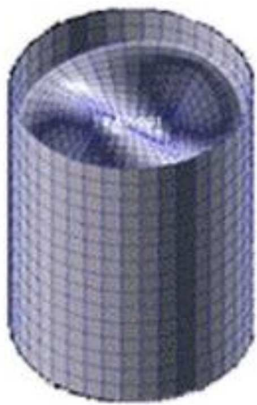

(d)

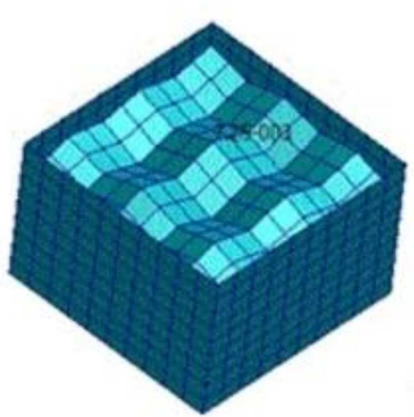

(h)

Fig. 1. (a) experimental result for the cylindrical tank $(d=200 \mathrm{~mm}$, mode $(1,1))$ with $f=1.71 \mathrm{~Hz}$, (b) virtual mass simulation for the cylindrical tank $(d=200 \mathrm{~mm}$, mode $(1,1))$ with $f=2.00 \mathrm{~Hz}$, (c) experimental result for the cylindrical tank $(d=200 \mathrm{~mm}$, mode $(1,2))$ with $f=3.04 \mathrm{~Hz},(\mathrm{~d})$ virtual mass simulation for the cylindrical tank $(d=200 \mathrm{~mm})$, mode $(1,2))$ with $f=3.63 \mathrm{~Hz}$, (e) experimental result for the rectangular tank $(a=150 \mathrm{~mm}, b=100$ mm, mode $(1,0))$ with $f=1.80 \mathrm{~Hz}$, (f) virtual mass simulation for the rectangular tank $(a=150 \mathrm{~mm}, b=100$ mm, mode $(1,0))$ with $f=2.06 \mathrm{~Hz}$, (g) experimental result for the square tank $(a=200 \mathrm{~mm}$, mode $(5,0))$ with $f=4.00 \mathrm{~Hz}$ (h) virtual mass simulation for the square tank $(a=200 \mathrm{~mm}$, mode $(5,0))$ with $f=4.70 \mathrm{~Hz}$.

A comparison of selected mode shapes from the laboratory experiments and virtual mass models are shown in the Fig. 1 [9]. The deviations of the virtual mass numerical solutions with respect to the analytical solutions of the Laplace equation are presented in Table 1. It should be emphasized that the derivation of the Laplace equation assumes a rigid tank container, whereas the virtual mass numerical solution takes the deformable plexiglass container into account. The sloshing frequencies of the experimental results, shown in Fig. 1a, c, e, g, were obtained by visual inspections at the start of the sloshing motions during the continuous frequency sweep operation of the shake table. Corresponding mode shapes obtained using the virtual mass method are presented in Fig. $1 b, d, f, h$.

The deviations between the modal frequencies increased with tank diameter but remained less than $15 \%$ for majority of cases. The deviations between the numerical and analytical results were consistently around 20 to $25 \%$ for the rectangular containers for all cases.

Meshing is an important issue which needs to be considered in the virtual mass method. A good mesh is essential for avoiding the ill conditioning of system matrices and accurately representing the problem domain.
Elements should not overlap and masses should be uniformly distributed. In addition, corners of the mesh should not be shared with a large number of elements and the elements should have low aspect ratios. The use of quad elements for meshing the rectangular liquid surface is straightforward but surface meshing of the circular surfaces are more challenging. Using a radial mesh causes a singularity on the central point and results in extreme differences in element aspect ratios. Two surface mesh types were evaluated for cylindrical tanks, namely, the butterfly mesh and the unstructured mesh. In order to compare these strategies, the free liquid surface of the cylindrical tank model with the diameter of $100 \mathrm{~mm}$ was meshed with a butterfly mesh with 500 elements and an unstructured mesh with 502 elements. The frequencies

\section{TABLE II}

The deviations of sloshing mode frequencies of virtual mass models from the theoretical values.

\begin{tabular}{c|c|c|c}
\hline \hline \multirow{2}{*}{$\begin{array}{c}\text { Mesh } \\
\text { type }\end{array}$} & 1st & 2nd & 3rd \\
\cline { 2 - 4 } & \multicolumn{3}{|c}{ frequency $[\mathrm{Hz}]$} \\
\hline butterfly & 2.84 & 2.84 & 3.49 \\
unstructured & 2.87 & 2.87 & 3.51
\end{tabular}




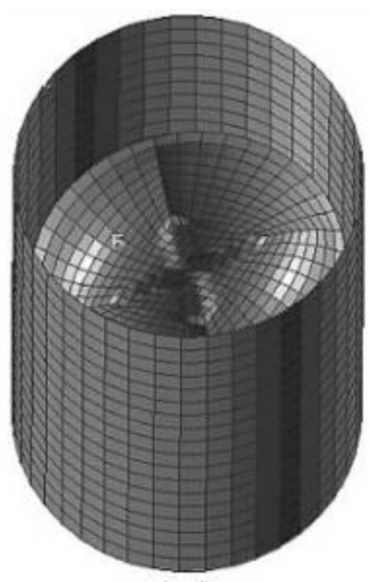

(a)

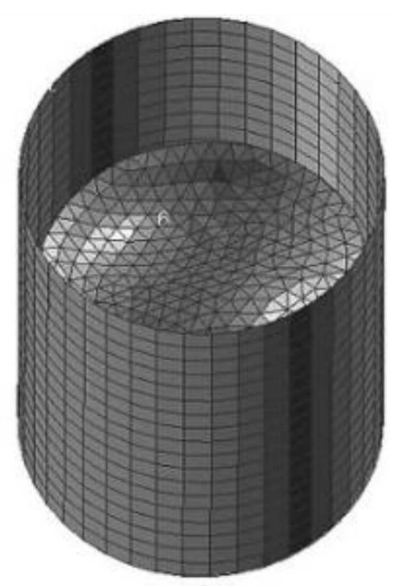

(b)
Fig. 2. First sloshing mode shape for (a) the butterfly mesh and (b) the unstructured mesh.

for the first three sloshing modes are presented in Table II and the mode shapes for the first mode are presented in Fig. 2a and b.

\section{Conclusions}

The virtual mass is an efficient method for analyzing the sloshing response liquid storage tanks. The deviations between the numerical and analytical values for the frequencies of sloshing modes were generally in the acceptable range. One of the major reasons for these deviations is the wall flexibility assumptions for the analytical solution and the numerical model. The analytical solution assumes that the wall is rigid whereas the virtual mass method takes into account the flexibility of the container wall.
Although the use of different meshing schemes did not have a profound effect on the vibration frequencies of sloshing modes for cylindrical tanks, the unstructured mesh topology provided more realistic results for the vibration mode shapes.

\section{Acknowledgments}

The second author is grateful to the funding provided by the Bogazici University Research Fund through Contract 07HT102. The authors acknowledge the assistance provided by Ceki Erginbas and Burak Tonga of the Graduate Program in Civil Engineering at Bogazici University

\section{References}

[1] A.K. Tang, Procedia Eng. 14, 922 (2011).

[2] P. Pal, S.K. Bhattacharyya, J. Sound Vib. 329, 4466 (2010).

[3] R.A. Ibrahim, Liquid Sloshing Dynamics: Theory and Applications, Cambridge University Press, New York 2005.

[4] O.M. Faltinsen, A.N. Timokha, Sloshing, Cambridge University Press, New York 2014.

[5] R.A. Ibrahim, J. Fluids Eng. 137, 090801 (2015).

[6] H.N. Abramson, The Dynamic Behavior of Liquids in Moving Containers NASA SP-106, National Aeronautics and Space Administration, Springfield 1966.

[7] K. Çakıroğlu, M.Sc. Thesis, Boğaziçi University, Istanbul 2012.

[8] MSC Nastran Advanced Dynamic Analysis Users Guide, MacNeal-Schwendler Corporation, Santa Ana 2004.

[9] S.A. Kilic, Sloshing effects in industrial cylindrical metal tanks and investigation of seismic behavior Bogazici University, Istanbul 2013, contr. 07HT102. 